9th A. Friedmann International Seminar and

3rd Casimir Symposium 2015

International Journal of Modern Physics: Conference Series

Vol. 41 (2016) 1660145 (8 pages)

(C) The Author(s)

DOI: $10.1142 /$ S2010194516601459

\title{
Ramsey resonance of coherent population trapping in slow rubidium beam
}

\author{
I. M. Sokolov \\ Department of Theoretical Physics, \\ Peter the Great St. Petersburg Polytechnic University, \\ Polytechnicheskaya 29, St.Petersburg, 295251, Russia, \\ IMS@IS12093.spb.edu \\ Received 14 September 2015 \\ Published 18 March 2016
}

\begin{abstract}
We calculate the coherent population trapping (CPT) resonance in slow beam of rubidium 87 atoms caused by of their interaction with bichromatic electromagnetic field in two separated spatial domains. In the case of monovelocity beam we study the properties of the CPT resonance depending on type of working transitions, velocity of the atomic beam, intensity and polarization of electromagnetic fields, and space separation in Ramsey scheme.

Keywords: Coherent population trapping; Ramsey method.

PACS numbers: 32.70.Jz, 32.80.Bx, 42.50.Gy
\end{abstract}

\section{Introduction}

The coherent population trapping (CPT) is a resonance effect caused by interaction of multimode radiation with multilevel atomic systems. ${ }^{1,2}$ In the simplest case CPT can be observed for three level atomic systems interacting with bichromatic laser field. If the detuning of the two laser frequencies satisfies the condition of twophoton resonance, atoms will be coherently trapped in the so called dark state, and will stop absorbing laser radiation. The width of CPT resonance is much less than natural linewidth and it gives opportunity to use this resonance in metrology and for creation of quantum frequency standards. ${ }^{3-5}$

Even more narrow resonance can be observed if CPT is registered by Ramsey method. ${ }^{6}$ For the first time CPT-Ramsey fringe was experimentally demonstrated for thermal atomic beam of $\mathrm{Na}$ atoms in Ref. 7. In a series of subsequent experiments the spatial separation of two areas of interaction of atoms with bichromatic

This is an Open Access article published by World Scientific Publishing Company. It is distributed under the terms of the Creative Commons Attribution 4.0 (CC-BY) License. Further distribution of this work is permitted, provided the original work is properly cited. 
radiation was replaced by their separation in the time domain. ${ }^{8,9}$ In Refs. 10,11 such experiments were performed with cold atoms confined in the atomic traps. The resonances with several Hertz width were observed. Narrowing of CPT resonance in the case of spatially zone pumping in atomic cells which is analog of Ramsey method were demonstrated in Refs. 12-14.

In Ref. 15 a scheme of a mobile slow-beam microwave atomic clock was proposed. This scheme does not assume usage of a bulky atomic trap. The slow atomic beam is generated from thermal one by means of a modified Zeeman slower. Utilization of the CPT resonance with Ramsey registration technique allows one to refuse from the microwave cavity and thus to decrease the size of the clock and its power consumption.

The main goal of the present work is to study some aspects of formation of the CPT signal in this atomic clock. To analyze the main regularities of this clock we restrict ourselves here by the model of monovelocity narrow atomic beam. We calculate time dynamics of atomic internal states in the beam and study the shape of the CPT resonance depending on the specific type of working transitions and polarization scheme. We consider also how the width and amplitude of resonance depends on such parameters as space separation in Ramsey scheme and the velocity of the atoms.

\section{Basic Assumptions and Approach}

Signal of a frequency discriminator based on the CPT effect is determined by varying of total population of atomic excited states in registration area under changes of two photon detuning. To calculate this population in the present paper we use the density matrix formalism. This formalism gives us opportunity to describe the interaction between atoms and laser radiation taking into account main factors responsible for generation the CPT-Ramsey signal.

Under typical conditions (see Ref. 15) the density of atoms in the beam does not exceeds $3 \times 10^{7} \mathrm{~cm}^{-3}$. For such a densities and for very small velocities of relative atomic motion we can neglect interatomic collisions. We can also neglect resonance dipole-dipole interatomic interaction which causes noticeable shifts and distortion of atomic transitions in cold gases. ${ }^{16-18}$ Thus when depicting the dynamics of atoms in the beam we can completely neglect interatomic interaction.

It is convenient to describe internal atomic dynamics in the co-moving frame of reference. The atomic motion for typical velocities can be considered classically. Momentum transmitted to the atom from the light in the result of their interaction is essentially smaller than both initial atomic longitudinal and transverse momentum in the beam. ${ }^{19}$ It allows us not to consider recoil effect.

In the co-moving frame of reference the atom is motionless. Its dynamic in external laser field is described by the following set of equations

$$
\frac{\partial \rho}{\partial t}=-\frac{i}{\hbar}\left[H_{0}+V, \rho\right]+\Gamma \rho .
$$


Here $\Gamma$ is spontaneous decay relaxation operator; $H_{0}$ is the Hamiltonian of free atom; $V$ is the operator describing atom-light interaction. In the dipole approximation

$$
V=-\mathbf{d} \mathbf{E}=-\mathbf{d}\left(\mathbf{e}_{1} E_{01}(t) \exp \left(-i \omega_{1} t\right)+\mathbf{e}_{2} E_{02}(t) \exp \left(-i \omega_{2} t\right)+\text { c.c. }\right) / 2 .
$$

Here we take into account that atomic ensemble interacts with two mode of the field which generally have different polarizations $\left(\mathbf{e}_{1}\right.$ and $\left.\mathbf{e}_{2}\right)$ and different frequencies $\left(\omega_{1}\right.$ and $\left.\omega_{2}\right)$. Note also that the spatial profile of the laser field is Gaussian and in considered frame the fields are essentially nonstationary. We indicate it explicitly by time dependence of both field amplitudes $E_{01}$ and $E_{02}$.

We will assume further that laser radiation is narrow enough and each mode of bichromatic field caused transitions from only one hyperfine sublevel of the ground state $F_{1}=I-1 / 2$ and $F_{2}=I+1 / 2$ of considered alkali atom (here $I$ is nuclear angular momentum).

In rotating wave approximation with regard to approximations discussed above the Eq. (1) has the following matrix form

$$
\begin{gathered}
\frac{\partial \rho_{g g^{\prime}}}{\partial t}=i\left(-\omega_{g g^{\prime}}-\left(\omega_{g}-\omega_{g^{\prime}}\right)\right) \rho_{g g^{\prime}}+\frac{i}{\hbar} \sum_{e}\left(-\tilde{V}_{g e} \rho_{e g^{\prime}}+\rho_{g e} \tilde{V}_{e g^{\prime}}\right)+(\Gamma \rho)_{g g^{\prime}} \\
\left.\frac{\partial \rho_{e g}}{\partial t}=i\left(-\omega_{e g}+\omega_{g}\right)\right) \rho_{e g}-\frac{i}{\hbar} \sum_{e}\left(\tilde{V}_{e g^{\prime}} \rho_{g^{\prime} g}\right)+(\Gamma \rho)_{e g} \\
\frac{\partial \rho_{e e^{\prime}}}{\partial t}=-i \omega_{e e^{\prime}} \rho_{e e^{\prime}}+\frac{i}{\hbar} \sum_{e}\left(-\tilde{V}_{e g} \rho_{g e^{\prime}}+\rho_{e g} \tilde{V}_{g e^{\prime}}\right)+(\Gamma \rho)_{e e^{\prime}} . \\
\tilde{V}_{e g}=\left\langle F_{e} M_{e}\left|d_{\mu}\right| F_{g} M_{g}\right\rangle e_{g}^{\mu} E_{0 g}(t) .
\end{gathered}
$$

Here $\omega_{j i}=\left(E_{j}-E_{i}\right) / \hbar ; \rho_{i j}$ are matrix elements of slow varying amplitude of the density operator. Indexes $g$ and $e$ correspond to ground and excite states respectively. These states are hyperfine ones and are characterized by quantum numbers $F$ and $M$. The frequency with one index $\omega_{i}$ denotes the frequency of that mode which causes transition from corresponding ground sublevel.

In general case the set of equations (3) takes into account all fine and hyperfine structure of atomic excited states. Further in calculation we restrict ourself by considering all hyperfine sublevel of only one D line. Corresponding equations are solved numerically for atomic beam having different velocities and, consequently, cross the laser rays over the different time intervals. This difference is taken into consideration by different dependencies $E_{01}(t)$ and $E_{02}(t)$. Obtained solution give us information about atomic state at any instant of time. Transforming this solution into laboratory system of coordinates we get information about atomic state at a given space point. In the following section we analyze the result of calculation in the case of rubidium 87 atomic beam.

\section{Results and Discussion}

Examination of CPT we begin with analysis of dynamics of dark states generation. Consider time evolution of atomic density matrix for the case when upper working 
level is $J=1 / 2 ; \quad F_{e}=2$; initial atomic state is $F_{g}=2$ and polarization scheme is lin $\perp$ lin. As we will show below this case corresponds to optimal scheme of observation of the $\mathrm{CPT}$ resonance.

Because the dynamics of internal atomic state in spatially inhomogeneous electromagnetic field is determined by speed of atoms it is convenient to show not dependence on the time but on the space coordinate of the atoms. Figure 1 demonstrates corresponding dependence for the atoms moving with speed $300 \mathrm{~cm} / \mathrm{c}$ perpendicular to the axes of light beams. The minimal distance from the axes is zero i.e. the atom passes through the region with maximal value of the field strength. The parameters of the light beams are the following: intensities of the both spectral components are equal to $10 \mu \mathrm{W} / \mathrm{cm}^{2}$ at the axes, Gaussian radii are $w=0.25 \mathrm{~cm}$. The axes are located at 2 and $32 \mathrm{~cm}$ from the exit of the Zeeman slower. The positions of the axes are shown in the figure by vertical reference lines. Magnetic induction of longitudinal (parallel to light rays) magnetostaticfield is $10 \mathrm{mG}$. These are typical parameters of studied standard. ${ }^{15,19}$ The calculations are performed for several different detuning of two-photon resonance.

In Fig. 1 we show two spatial areas of the atomic trajectory. The first one includes tree initial centimeters of the path. Here the atomic beam interacts with radiation for the first time and here the dark state is generated. The second section corresponds to tree centimeters near registration domain. Between these two areas the atoms do not interact with electromagnetic field and their states do not change therefore this part of trajectory is nor shown.

Figure 1a clearly indicates nonmonotonic dependence of atomic excitation on position of the atom in both areas of light-atom interaction. Initially the population of upper level increases. In the course of time interacting with bichromatic field atoms pass into dark state which is coherent superposition of several Zeeman sublevels of the ground state. This fact is well illustrated by Fig. 1b. Being in the dark state atoms do not interact with the radiation. The population of excited sates
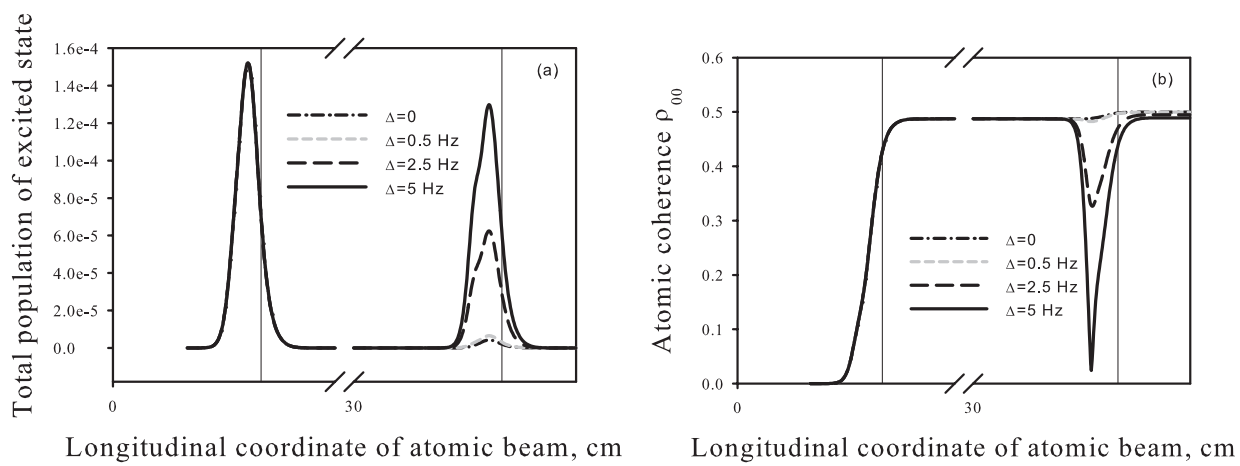

Fig. 1. Dynamics of total population of excited state (a) and atomic coherence $\rho_{00}$ (b) for different detuning of two photon resonance. Vertical reference lines show the location of axes of the light beams. 
becomes nearly zero. Under considered conditions the dark state is populated before atoms reach the axis of light beam. Note also that for two-photon detuning which does not exceed several Hertz different curves are undistinguished in the first area.

Leaving the first light beam the atoms evolve as free particles. The small remaining population of upper level decreases up to zero due to spontaneous decay. The hyperfine coherence of the ground state oscillates at the frequency of free atomic transition. Its absolute value does not change. The type of processes which take place in the area of registration depends on the value of two-photon detuning. If it is zero the frequency of free oscillations is equal to difference of frequencies of the two components of bichromatic field. In such a case the state of the atom at the entrance of registration zone is still dark for given field. Insignificant increasing of $\rho_{00}$ connects with the fact that small part of the atoms are in the bright state here.

If two-photon detuning is not zero the transient processes take place. During these processes the new dark state is generated. Created coherence oscillates at the difference frequency of two modes of the radiation. Absolute value of hyperfine atomic coherence decrease initially, then it restores (see Fig. 1b). Increasing of twophoton detuning leads to increasing in excited state population and consequently to discriminator signal enhancement. We however are interested not in maximal value of signal in registration zone of Fig. 1. Area under curve is more important parameter. This area determines the total signal caused not by all atoms in the registration zone.

Figure 1 is calculated for one possible scheme of registration. Proper choice of working atomic transitions and polarizations of two components of used bichromatic radiation is the key point in construction of registration scheme of the CPT standard. In suggested beam standard all atoms after Zeeman slower are on one hyperfine sublevels $F_{g}$ of the ground state. Typically for ${ }^{87} \mathrm{Rb}$ it is $F_{g}=2$ but one can get beam in $F_{g}=1$ state. Besides that in considered device narrow-band laser is assumed to be used. For very small velocity of the atomic beam and its small angular divergence of about 1/100 rad the hyperfine structure of excited state is well resolved. It means that we can choose specific hyperfine sublevel of excited state as working one. It can belong to $D_{1}$ or $D_{2}$ multiplet. As for polarization scheme, the CPT resonance can be observed both in circularly and linearly polarized waves. ${ }^{5,8,20-22}$ However earlier studies have shown that circularly polarized light has definite disadvantage. According to selection rules there are so called "trap" states for this polarization. Part of atoms accumulates in these traps due to optical pumping and does not create ground states coherence. It decreases the contrast of the CPT resonance. For this reason we will not consider circularly polarized light in this work. Examining only the case of linear polarization and taking into account two initial atomic states and all possible atomic transitions in $D_{1}$ or $D_{2}$ lines we have sixteen different alternative schemes of registration.

To find the best option we made numerical comparison of all these sixteen schemes. Figure 2 demonstrates corresponding comparison for the same parameters as were used for Fig. 1. The CPT resonance was calculated assuming that frequency 

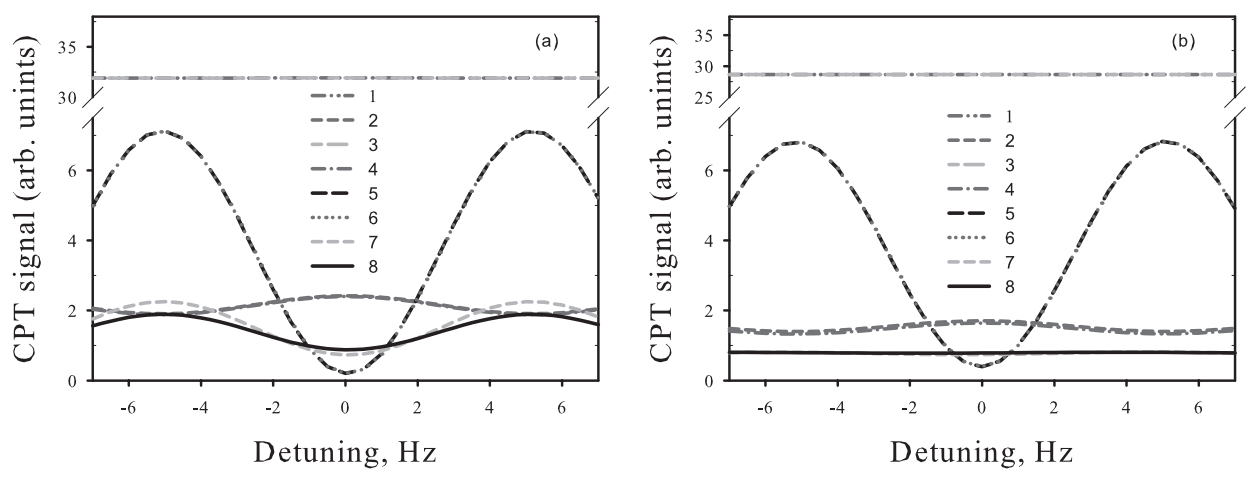

Fig. 2. CPT singal for different conditions (a) $D_{1}$ line; (a) $D_{2}$ line; $1-1-4 \operatorname{lin} \| \operatorname{lin} ; 5-8-\operatorname{lin} \perp \operatorname{lin} ; 1$ $-F_{g}=2, F_{e}=2 ; 2-F_{g}=2, F_{e}=1 ; 3-F_{g}=1, F_{e}=2 ; 4-F_{g}=1, F_{e}=1 ; 5-F_{g}=2, F_{e}=2$; $6-F_{g}=1, F_{e}=2 ; 7-F_{g}=2, F_{e}=1 ; 8-F_{g}=1, F_{e}=1$.

of light applied to transition $F_{2} \leftrightarrow F_{e}$ is in exact resonance and two photon detuning is created by scanning of frequency of radiation applied to the second, $F_{1} \leftrightarrow F_{e}$ transition. Signal in Fig. 2 corresponds to the number of photons of spontaneous fluorescence for unit total flux of atoms in the beam.

In Fig. 2 we show all sixteen possible CPT resonances for linearly polarized light. Some pairs of resonance curves coincide exactly with each other. Some pairs of curves are very close. Curves in these pairs correspond to different initially populated atomic ground hyperfine sublevel. It means that choice of this sublevel does not allow us to influence essentially the figure of merit of the beam standard. At the same time in Zeeman slower cyclical transition $F_{g}=2 \rightarrow F_{e}=3$ is used and atomic transition to $F_{g}=1$ state is compensated by auxiliary laser. So without special effort after Zeeman slower one gets the atoms in $F_{g}=2$ state. Further we will restrict our consideration by this case.

The maximal contrast is observed for resonance tuned at excite $F_{e}=2$ levels of $D_{1}$ line for orthogonal linearly polarized waves. Polarization schemes with parallel polarization give resonances with noticeably smaller contract. It connects with specific selection rules and with signs of Clebsh-Gordon coefficients.

CPT resonances for $D_{2}$ line (see Fig. 2b) have similar peculiarities as for $D_{1}$ line. Theirs amplitudes are close but contrasts are smaller, so $D_{1}$ line is preferable for observation CPT effects. Note that the main results have calculated for beam Ramsey registration scheme are coincide qualitatively with known results for steady state CPT in gas cells. ${ }^{5,8,20-22}$

In Fig. 2 we show only one resonance from Ramsey fringe. The period of this fringe as well as width of Ramsey resonances are determined by the delay time between generation of dark state and its registration. They decrease with this time. This fact can be seen in Fig. 3 . 

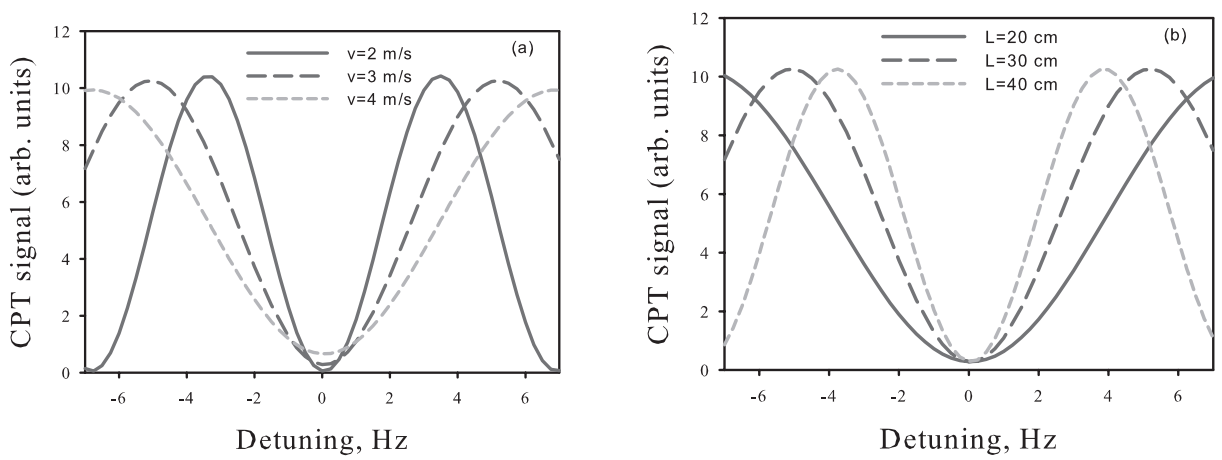

Fig. 3. (a) Dependence of the CPT signal on velocity of atomic beam, space separation in Ramsey scheme $L$ is $30 \mathrm{~cm}$. (b) Dependence of the CPT signal on space separation in Ramsey scheme L, velocity of atomic beam is $300 \mathrm{~cm} / \mathrm{s}$.

In Fig. 3 we show the CPT-Ramsey resonances for different velocities of the atoms (Fig. 3a) and for different space separation in Ramsey scheme (Fig. 3b). In all considered cases the width increases proportional to space separation and inversely to the velocity. For small velocities we see some additional contrast increasing because for relatively big velocities not all atoms transit into dark state while cross the light beams.

\section{Conclusion}

In the present work we calculated the time dynamic of atomic internal state in the beam interacting with two spatially separated region of bichromatic field. We analyze the formation of dark state and influence of two-photon detuning on CPT resonance registration process.

We compare the CPT-Ramsey signal for all possible scheme of working transitions in $D_{1}$ and $D_{2}$ lines for linearly polarized light for $\operatorname{lin} \| \operatorname{lin}$ and $\operatorname{lin} \perp \operatorname{lin}$ cases. The calculation shows that the most contrast signal is observed for $\operatorname{lin} \perp \operatorname{lin}$ if two components of bichromatic field are tuned at sublevel $F_{e}=2$ of $D_{1}$ multiplet.

\section{Acknowledgments}

We acknowledge financial support from the Ministry of Education and Science of the Russian Federation (State Assignment 3.1446.2014K).

\section{References}

1. B. D. Agap'ev, M. B. Gornyj, B. G. Matisov and Yu. V. Rozhdestvenskii, Physics-Usp. 36, 763 (1993).

2. E. Arimondo, in Progress in Optics, ed. E. Wolf (Elsevier, Amsterdam, 1996), Vol. XXXV, p.257. 
3. J. Kitching, S. Knappe and L. Hollberg, Appl. Phys. Lett. 81, 553 (2002).

4. S. Knappe, V. Shah, D. Peter, D. Schwindt, L. Hollberg, J. Kitching, L. Liew and J. Moreland, Appl. Phys. Lett. 85, 1460 (2004).

5. J. Vanier, Appl. Phys. B 81, 421 (2005).

6. N. F. Ramzey, Phys. Rev. 78, 695 (1950).

7. J. E. Thomas, P. R. Hemmer, S. Ezekiel, C. C. Leiby, Jr., R. H. Picard and C. R. Willis, Phys. Rev. Lett. 48, 867 (1982).

8. T. Zanon, S. Guerandel, E. de Clercq, D. Holleville, N. Dimarcq and A. Clairon, Phys. Rev. Lett. 94, 193002 (2005).

9. G. Pati, K. Salit, R. Tripathi and M. Shahriar, Opt. Commun. 281, 4676 (2008).

10. C. Xi, Y. Guo-Qing, W. Jin and Z. Ming-Sheng, Chin. Phys. Lett. 27, 113201 (2010).

11. F. X. Esnault, E. Blanshan, E. N. Ivanov, R. E. Scholten, J. Kitching and E.A. Donley, Phys. Rev. A 88, 042120 (2013).

12. Y. Xiao, I. Novikova, D. F. Phillips and R. L. Walsworth, Opt. Express 16, 14218 (2008).

13. E. Breschi, G. Kazakov, C. Schori et. al., Phys. Rev. A 82, 063810 (2010).

14. G. Kazakov, A. Litvinov and B. Matisov, Quantun Electronics 42, 185 (2012).

15. V. S. Zholnerov, A. K. Vershovskiy and Yu. V. Rozhdestvenskiy, Project of a satellite slow beam atomic clock with CPT-Ramsey registration, in Proc. 2012 European Frequency and Time Forum (Gothenburg, Sweden, 2012), p. 320.

16. I. M. Sokolov, D. V. Kupriyanov, R. G. Olave and M. D. Havey, J. Mod. Opt. 57, 1833 (2010).

17. Ya. A. Fofanov, A. S. Kuraptsev, I. M. Sokolov and M. D. Havey, Phys. Rev. A 84, 053811 (2011).

18. A. S. Kuraptsev and I. M. Sokolov, Phys. Rev. A 90, 012511 (2014).

19. I. M. Sokolov, Registration of coherent population trapping resonance in rubidium 87 beam by means of Ramsey method, to appear in Quantum Electronics.

20. Y.-Y. Jau, E. Miron, A. B. Post, N. N. Kuzma and W. Happer, Phys. Rev. Lett. 93, 160802 (2004).

21. A. V. Taichenachev, V. I. Yudin, V. L. Velichansky and S. A. Zibrov, JETP Letters 82, 398 (2005).

22. G. A. Kazakov, B. G. Matisov, I. E. Mazets, G. Mileti and J. Delporte, Phys. Rev. A 72, 063408 (2005). 